C-A/AP/\#412

Nov. 2010

BNL-94373-2010-IR

\title{
Emittance Measurements using Vernier Scans During Run 09 (pp at $250 \mathrm{GeV}$ )
}

\author{
A. Drees
}

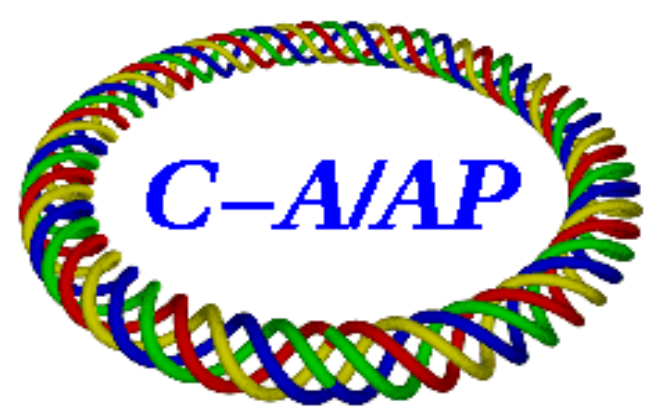

\section{Collider-Accelerator Department}

Brookhaven National Laboratory

Upton, NY 11973

Notice: This document has been authorized by employees of Brookhaven Science Associates, LLC under Contract

No. DE-AC02-98CH10886 with the U.S. Department of Energy. The United States Government retains a non-

exclusive, paid-up, irrevocable, world-wide license to publish or reproduce the published form of this

document, or allow others to do so, for United States Government purposes. 


\title{
Emittance Measurements using Vernier Scans during Run 09 (pp at $250 \mathrm{GeV}$ )
}

\author{
A. Drees
}

November 15, 2010

\section{Vernier Scan Technique}

During the $250 \mathrm{GeV}$ PP run in 09 a total of 9 vernier scans [1] were performed. The transverse size and shape of the beam overlap region is measured by recording the relative interaction rates as a function of the transverse beam separation. A fit of the measured interaction rates as a function of the separation will allow to determine the effective beam size as well as the maximum achievable collision rate and the effective cross section (see eq. 2). In reality additional effects such as crossing angle or hourglass effect [2] require correction factors to be applied and will have to be taken into account when computing the systematic error. Table 1 lists the fills with vernier scans together with the hour glass factors achieved at the time of the vernier scans, the fill pattern and the number of colliding bunch pairs in IR8 and IR6. Some fills include scans in both IR's while others include a scan in one IR only. Either way, however, each of those fills will give us a beam emittance measurement.

\begin{tabular}{|l|c|c|c|c|c|}
\hline fill & hourglass factor & IR & fill pattern & \# coll. pairs IR8 & IR6 \\
\hline 10207 & 0.72 & IR6\&8 & $54 \times 56$ & 54 & 50 \\
10276 & 0.64 & IR6\&8 & $56 \times 56$ & 56 & 52 \\
10325 & 0.64 & IR8 & $84 \times 84$ & 82 & 77 \\
10399 & 0.68 & IR6 & $83 \times 83$ & 80 & 75 \\
10415 & 0.68 & IR6\&8 & $109 \times 108$ & 106 & 101 \\
10478 & 0.70 & IR8 & $109 \times 107$ & 105 & 100 \\
10505 & 0.65 & IR8 & $109 \times 106$ & 104 & 99 \\
10507 & 0.73 & IR6 & $108 \times 106$ & 103 & 98 \\
10536 & 0.72 & IR6\&8 & $106 \times 106$ & 101 & 96 \\
\hline
\end{tabular}

Table 1: List of fills with vernier scans during the $250 \mathrm{GeV}$ '09 proton run.

The definition of luminosity assumes perfectly Gaussian beams. In hadron machines, where the damping is very low, this is not always the case and non-Gaussian tails can appear. This was first observed in RHIC during the $250 \mathrm{GeV}$ pp run ([3]). These nonGaussian components of the beam still contribute to the overall luminosity and have to be taken into account while computing the overlap area width. 


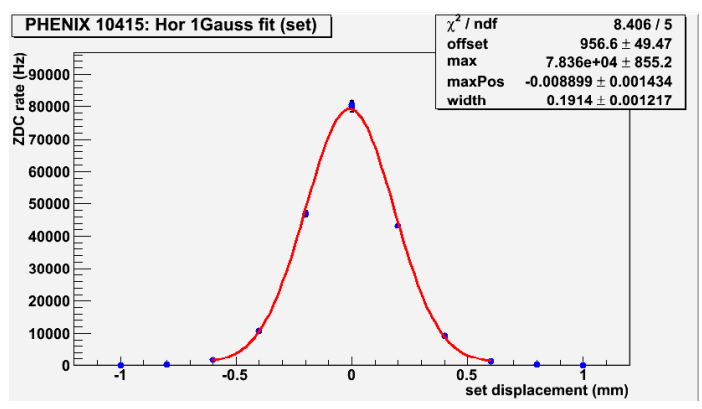

Figure 1: Horizontal beam profile as measured from the ZDC in PHENIX during the 10415 scan. A simple 1-Gauss-fit, truncated at $\pm 0.5 \mathrm{~mm}$, (red line) is applied.

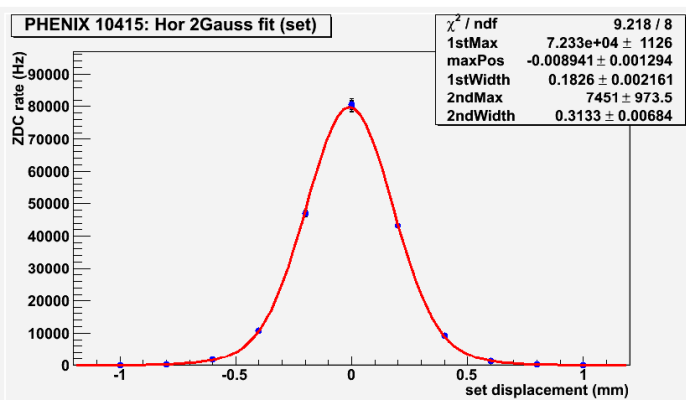

Figure 2: Horizontal beam profile as measured from the ZDC in PHENIX during the 10415 scan. A double Gauss-fit (red line) is applied.

The core of the beam, which remained Gaussian during run09, is the main contributor to the luminosity. A convenient way to include the tails in the model is to fit the profile with a double Gaussian. Figure 1 shows a fit to the data from store 10415 (PHENIX). Here a 1-Gauss fit, truncated to $\pm 0.5 \mathrm{~mm}$ is shown, resulting in a width of the overlap region of $0.19 \mathrm{~mm}$.

Figure 2, however, shows a fit to the same data as shown in figure 1 to the left, but applying a double Gauss fit. The resulting widths are $0.18 \mathrm{~mm}$ and $0.31 \mathrm{~mm}$ respectively. These widths are combined to an effective width $\sigma_{\text {eff }}$ according to:

$$
\sigma_{e f f}=\frac{1 s t W i d t h * 1 s t M a x+2 n d W i d t h * 2 n d M a x}{1 s t M a x+2 n d M a x}
$$

Combining the widths from the given example and using their individual relative maxima $(72330 \mathrm{~Hz}$ and $7451 \mathrm{~Hz}$ respectively) we get an effective width of $0.195 \mathrm{~mm}$, which is in good agreement with the result from the truncated 1-Gauss fit (figure 1). This indicates that the contribution from the second Gauss to the effective width is small and not significant although the two fits disagree obviously in the tail area as can easily be seen in figure 3. Comparing the two different fits as shown in figure 3 for another example, here fill 10536, demonstrates that a 1-Gauss fit does not describe the tails of the data while a double Gauss does. Both, however, fit the core portion of the profile well. Since both methods, truncating the 1-Gauss approach as well as the double Gauss fit to the whole range of separation, give similar results, they could both be used to determine the beam emittance. In the following we have combined the results from both methods while adding the range of variation between the two methods as a systematic error resulting in an overall $\pm 2 \pi \mathrm{mm}$ mrad error. However, the variation of the individual results per fill is typically small and in the order of $5 \%$ or less.

\section{Emittance Measurements}

\subsection{Vernier Scans}

From the fit to the vernier scan data the RMS beam size $\sigma_{x, y}$ as well as the effective cross section $\sigma_{p p}^{Z D C}$ of a detector, in this case the ZDCs, can be derived:

$$
\sigma_{p p}^{Z D C}=\frac{R_{\max } 2 \pi n_{B} n_{Y} \sigma_{x}^{V S} \sigma_{y}^{V S}}{n_{\text {coll }} f_{\text {rev }} N_{B} N_{Y}}
$$




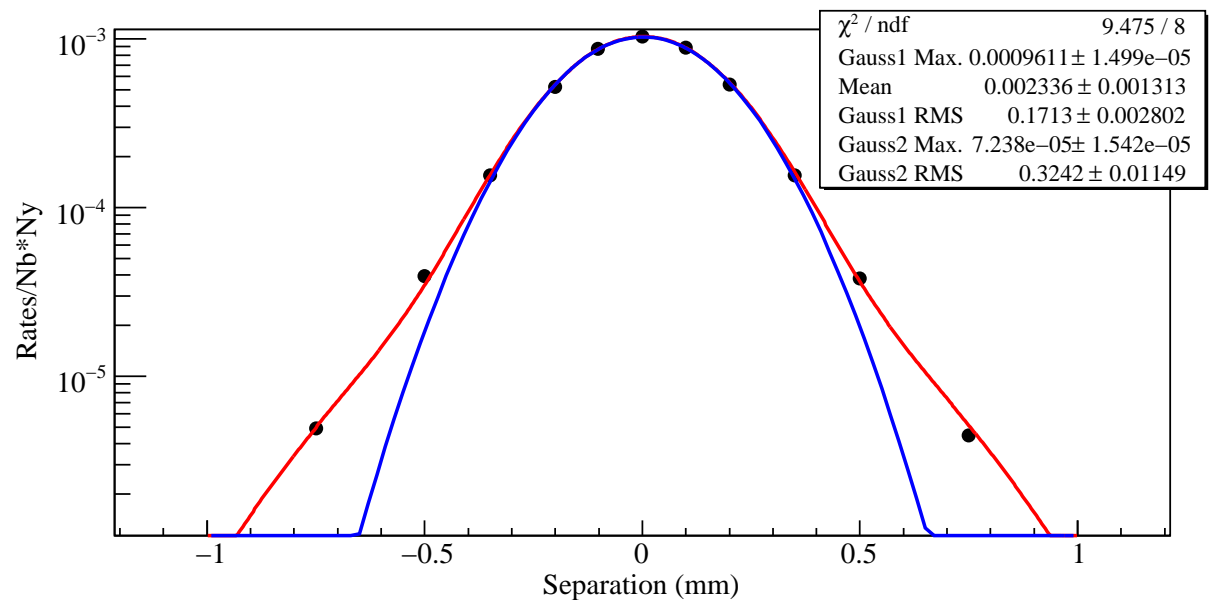

Figure 3: Profile measured by the ZDCs in PHENIX during the 10536 store showing both, a simple 1-Gauss fit (blue line) and a double Gauss fit (red line). Results from the double Gauss fit are included. The collision rate is normalized by the total beam current.

where:

$R_{\text {max }}=$ maximum collision rate seen by the ZDC detector (corrected for background)

$n_{B}, n_{Y}=$ number of blue and yellow bunches respectively

$\sigma_{x, y}^{V S}=$ RMS beam-overlap size, derived from the fit to the vernier scan data

$n_{\text {coll }}=$ number of colliding bunch pairs in the IP where the ZDC detector is located

$f_{\text {rev }}=$ revolution frequency, $78.4 \mathrm{kHz}$

$N_{B, Y}=$ total number of blue and yellow protons

Given the RMS beam size we can calculate the normalized beam emittance $\epsilon_{\text {norm }}^{x, y}$ assuming that the beam sizes are the same in the two rings:

$$
\epsilon_{\text {norm }}^{x, y}=\frac{6\left(\sigma_{x, y}^{V S}\right)^{2} \gamma}{2 \beta^{*}}
$$

with $\gamma=266.3$ for $250 \mathrm{GeV}$ protons.

\section{$2.2 \quad$ IPM}

At the same time the IPM detectors provide an equivalent measurement, not only at specific times but continuously during the store. As can be seen in figure 4, top, a constant is a good fit to the emittance as a function of time for the time span of 30 minutes (the duration of the vernier scan, shown in the bottom graph). The conclusion from figure 4 applies to all stores with vernier scans. There is practically no visible slope in the emittance data during the times of the vernier scans. Also typical are the differences between the rings and planes as apparently seen by the IPM. The results from the IPMs are listed together with the results from the vernier scans and from the collision rate ([4]) in table 2 below. The typical scatter of the IPM measurements indicates an error of about $3 \pi \mathrm{mm}$ mrad, which was used in the following.

\subsection{Emittance from Collision Rate}

In order to compute the beam size from the collision rate an effective cross section is needed. Combining eq. 2 and eq. 3 from above, one can compute the normalized emittance 

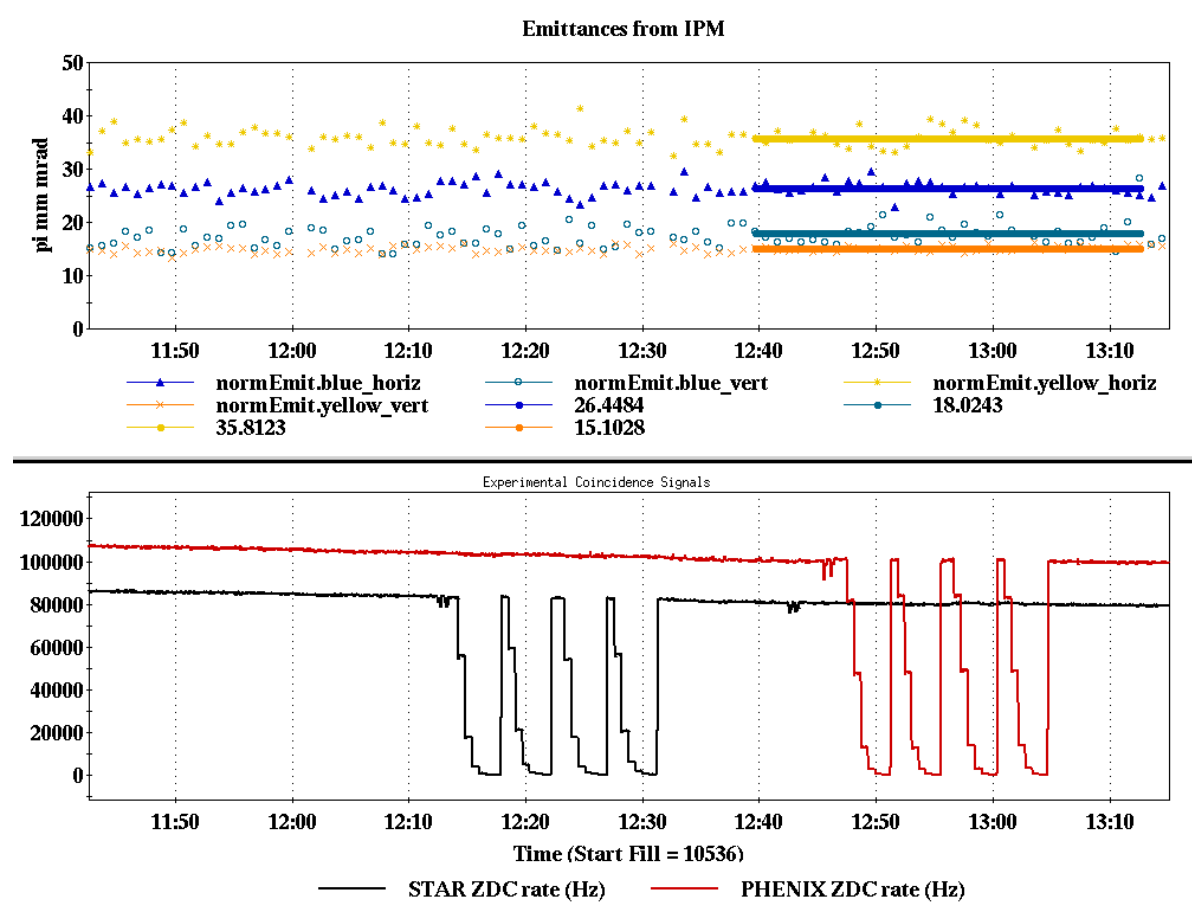

Figure 4: Emittance measurements from the IPM detector during the vernier scans in IR6 and IR8, fill 10536. Top: IPM data, bottom: ZDC coincidence rates in STAR and PHENIX. A constant fit was applied to the IPM data resulting in normalized emittances of $26,18,36$ and $15 \pi \mathrm{mm}$ mrad at the time of the vernier scan in PHENIX.

$\epsilon_{\text {norm }}^{\text {coll from the collision rate: }}$

$$
\epsilon_{\text {norm }}^{\text {coll }}=\frac{6 \gamma}{\beta^{*}} \frac{\sigma^{Z D C} n_{\text {coll }} f_{\text {rev }} N_{B} N_{Y}}{R_{\text {coll }} 2 \pi n_{B} n_{Y}}
$$

For this to work the following assumptions were made: the beam size in the two rings and both planes are effectively identical, beams are colliding head-on, $\beta^{*}=0.7 \mathrm{~m}$ and we know the effective cross section. In this analysis two values, 2.1 and 2.4 mbarn, were used as $\sigma^{Z D C}$ and the mean value is used in the following (referred to as "StoreAna" in table 2) with the average spread as the error bars shown in fig. 5 and 6 .

\section{Discussion}

All fit results are summarized in table 2. The fit results from the double Gauss fit are all slightly larger than the results from the truncated simple Gauss fit. This is consistent with an effective beam size which is, however mildly, somewhat enlarged due to the nonGaussian tails. However, the differences between fit functions as well as the two planes have the same order of magnitude as the error bar of $\pm 2 \pi \mathrm{mm}$ mrad. The results from the fits to the vernier scan data clearly show that we have round beams, hence the various results are all combined into one, labeled "Avg.".

On the other hand, the IPM data, shown in the last 4 columns, are consistent with beams that are larger in the horizontal plane than in the vertical one by about a factor 2-3 (depending on the ring). Therefore they were not averaged into one number and could not easily be compared with the emittance derived from collision rates (1st column). The results presented in table 2 are depicted in figure 5 and 6 . 


\begin{tabular}{|l||c||c|c|c|c|c||c|c|c|c|}
\hline \multicolumn{1}{|c||}{ fill } & \multicolumn{1}{c||}{ StoreAna } & \multicolumn{3}{c||}{ Vernier Scans } & \multicolumn{1}{c||}{ Avg. } & \multicolumn{4}{c|}{ IPM } \\
\hline & & $\epsilon_{H}^{1 G}$ & $\epsilon_{V}^{1 G}$ & $\epsilon_{H}^{2 G}$ & $\epsilon_{V}^{2 G}$ & & BH & BV & YH & YV \\
\hline 10207 & 44.5 & 42.5 & 45.5 & 43.0 & 46 & 44 & 61 & 43 & 34 & 18 \\
10276 & 31 & 28 & 29.5 & 29 & 31 & 29 & 44 & 34 & 45 & 14 \\
10325 & 19 & 20.5 & 22 & 22 & 23 & 22 & 29 & 22 & 33 & 13 \\
10399 & 21 & 20 & 19 & 21 & 20 & 20 & 24 & 19 & 29 & 12 \\
10415 & 30.5 & 29 & 29 & 30 & 29 & 29 & 33 & 26 & 40 & 17 \\
10478 & 19 & 18 & 21 & 18 & 20 & 19 & 23 & 14 & 26 & 11 \\
10505 & 20.5 & 18 & 21.5 & 19.5 & 21.5 & 20 & 25 & 16 & 31 & 12 \\
10507 & 18 & 19 & 18 & 19 & 18 & 18 & 23 & 12 & 27 & 11 \\
10536 & 26.5 & 22 & 24 & 24 & 25 & 24 & 26 & 18 & 36 & 15 \\
\hline
\end{tabular}

Table 2: Normalized beam emittance derived from collision rates [4], vernier scans and IPM detector in units of $\pi \mathrm{mm}$ mrad. In this analysis we assume $\beta^{*}=0.7 \mathrm{~m}$.

Fig. 5 top shows the individual fit results from the vernier scan data (both planes, both fit functions). The results from the 1-Gauss fit are shown in red, the results from the double Gauss fit are shown in green. Black data points represent the effective beam size computed from the collision rate according to eq. 4 . The agreement between the two fitting approaches as well as the methods is very good, all give basically the same answer. There is no systematic discrepancy between the two methods indicating that a $\beta^{*}=0.7 \mathrm{~m}$ is a good approximation for the 'true' value since no a priori knowledge of $\beta^{*}$ is needed in the vernier scan analysis. The measurements are all consistent with round beams and emittances between 18 and $45 \pi \mathrm{mm}$ mrad. The bottom plot compares the emittance from averaged vernier scan results (green data points, "VS ave.") with the data from the IPM (obviously labeled). The disagreement is quite large.

Figure 6 compares the IPM data from the horizontal (top) and vertical (bottom) plane with the vernier scan data separately. The top graph presents the horizontal emittance measurements from the double Gauss fit to the vernier scan data ("2GH Vscan") together with the blue and yellow IPM measurements in the same plane. The equivalent for the vertical plane is shown in the bottom graph. In both planes the disagreement with the vernier scan data is quite large. However, beginning with fill 10325, the blue IPM agree much better with the vernier scan data though the horizontal measurements are systematically above and the vertical measurements are systematically below the vernier scan results. This discrepancy is much more pronounced with the yellow IPM measurements which are clearly inconsistent with a round-beam assumption. Parts of that discrepancy could probably be explained by an uncertainty of the beta function at the location of the IPM. If so, the ratio between the two methods would, if not be 1 , be constant and not vary from fill to fill. Ignoring the first two data sets, which seem to be particularly off, the ratio between the two methods (VS versus IPM) varies between 0.77 and 0.91 in Blue horizontal and between 0.63 and 0.75 in Yellow. Therefore there is a $25 \%$ to $35 \%$ disagreement that is not quite constant but varies within $20 \%$ from fill to fill. The picture is similar in the vertical plane. In Blue the ratios vary from 1.04 to 1.46 with a $30 \%$ fill to fill scatter. In Yellow, where the disagreement with up to $80 \%$ between the methods is the largest, the 


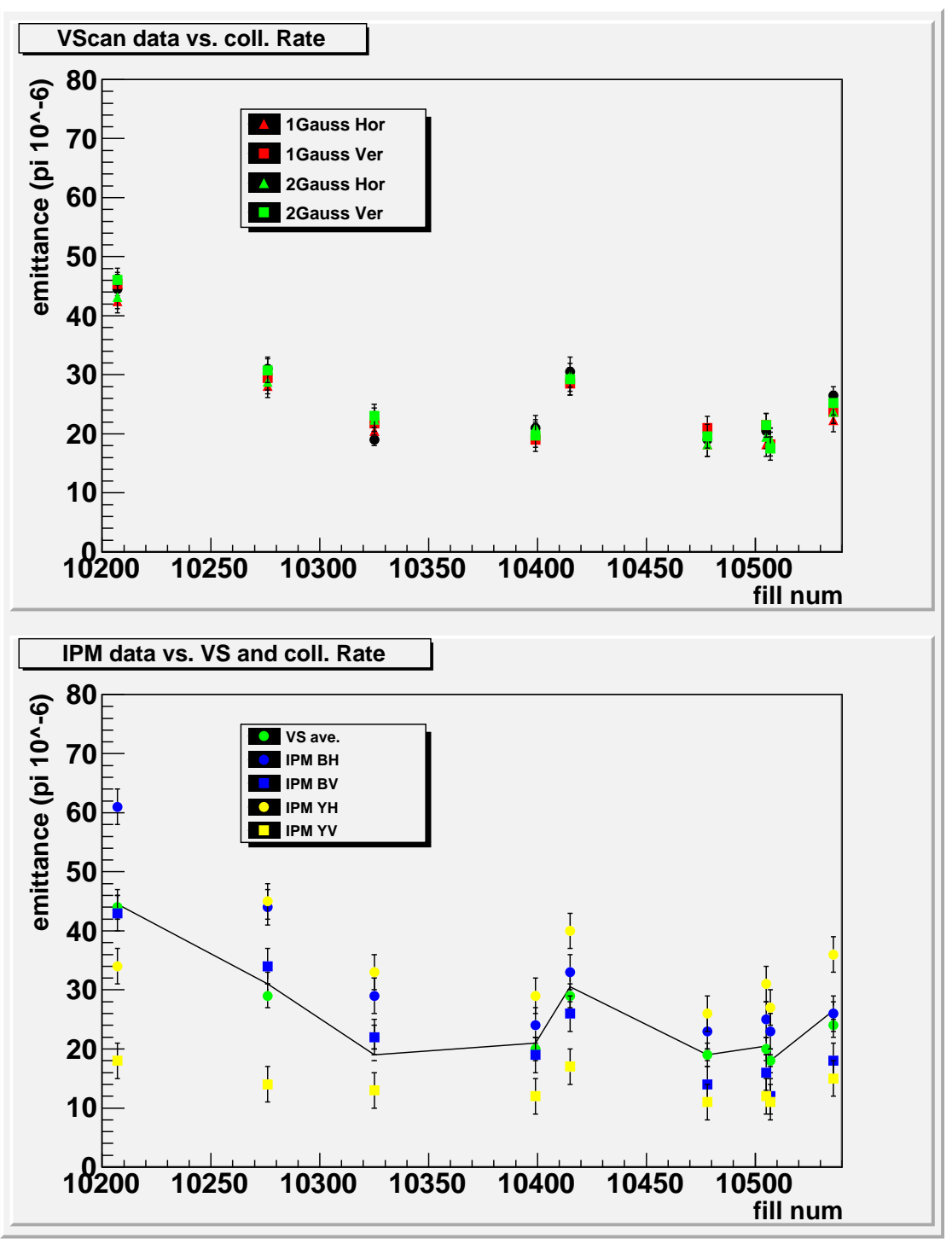

Figure 5: Emittances from vernier scans and collision rate during Run09 pp at $250 \mathrm{GeV}$ vs. fill number.

ratio ranges from 1.59 to 1.79 with a $10 \%$ fill to fill scatter.

\section{Conclusion}

Emittance measurements using the vernier scan technique give reliable results for $250 \mathrm{GeV}$ protons even though the transverse beam profiles have non-Gaussian tails. Those nonGaussian tails were observed for the first time this run at the $250 \mathrm{GeV}$ beam energy.

The vernier scan measurements are in excellent agreement with the emittances derived from collision rates and show practically no fill to fill scatter if compared to the latter. The results are consistent with a $\beta^{*}$ of $0.7 \mathrm{~m}$ and round beams.

The IPM measurements show a discrepancy of up to $80 \%$ compared with the vernier scan data and a fill to fill scatter of up to $30 \%$. If an uncertainty in the beta-function at the location of the IPM is the root cause, this uncertainty seems to be quite large. In any case, such an uncertainty could not explain the fill to fill variations of up to $30 \%$ which indicate 


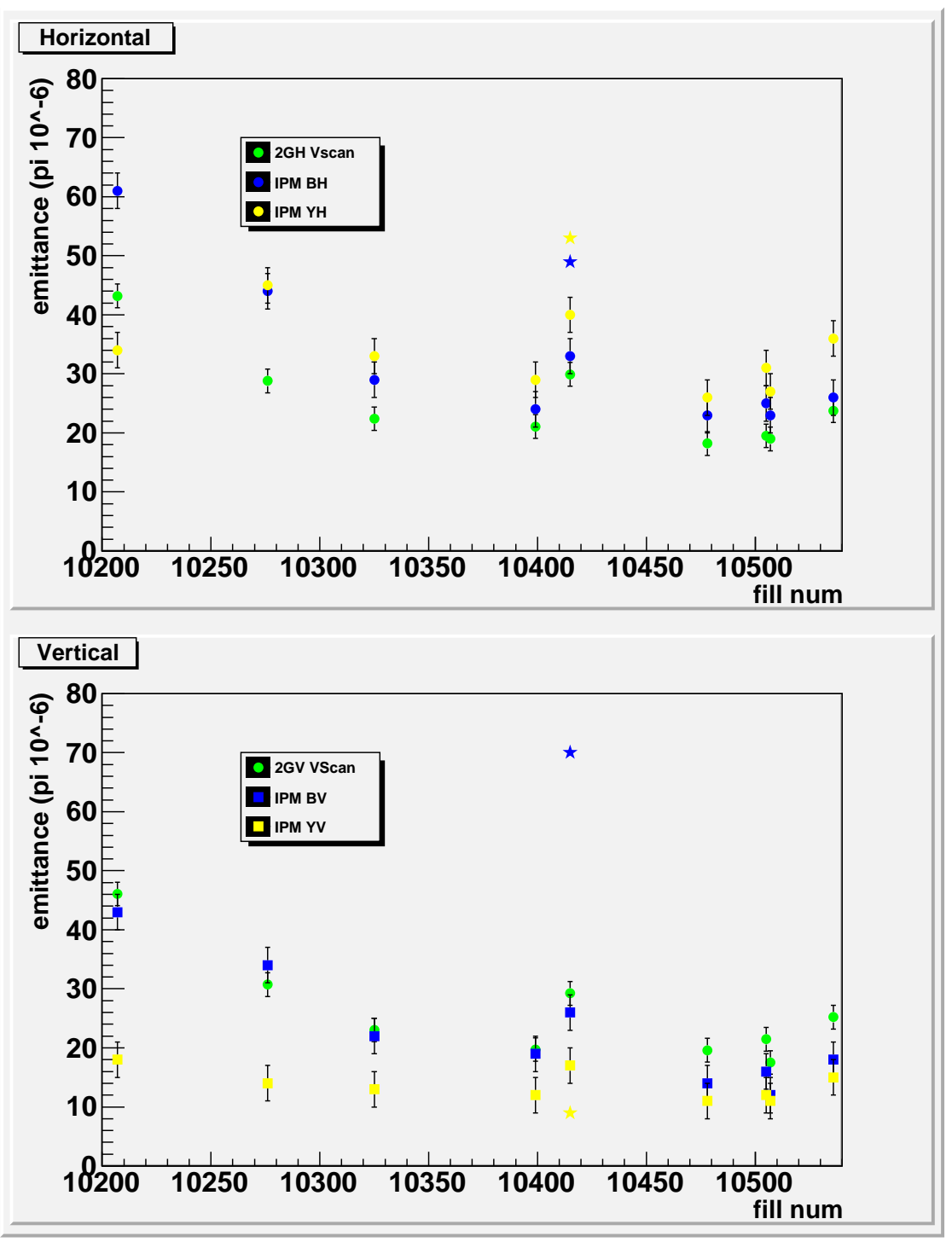

Figure 6: Horizontal (top) and vertical (bottom) normalized emittance measurements as a fct. of fill number combining data from vernier scans and IPM.

yet another underlying reason that could explain fill to fill variations (candidates could be beam intensity issues with the IPM, beam position at the IPM, varying background etc.).

\section{References}

[1] S. Van Der Meer, ISR-PO/68-31, KEK68-64.

[2] M. A. Furman, "Hourglass Effects for Asymmetric Colliders",PAC Proceedings", 1991.

[3] A. Drees, S.M. White, "Vernier Scan Results from the First RHIC Proton Run at 250 GeV", IPAC Proceedings, 2010.

[4] Using the StoreAnalysis program, courtesy W. Fischer and Sev Binello, RHIC operations software 